RAIRO Operations Research

RAIRO Oper. Res. 37 (2003) 213-219

DOI: $10.1051 /$ ro:2004007

\title{
ENVELOPPE CONVEXE DES HYPERPLANS D'UN ESPACE AFFINE FINI
}

\author{
Olivier Anglada ${ }^{1}$ et Jean François Maurras ${ }^{1}$
}

\begin{abstract}
In this paper we characterize by the facets the convex hull of the characteristic vectors of the hyperplanes of a finite projective space and of a finite affine space.
\end{abstract}

Résumé. Dans cet article nous caractérisons, par les facettes, l'enveloppe convexe des vecteurs caractéristiques des hyperplans d'un espace projectif fini et d'un espace affine fini.

\section{INTRODUCTION}

Un espace projectif fini d'ordre $q$ et de dimension $d$ est un objet $\mathcal{E P}$ constitué d'un ensemble $E$ de $q^{d}+q^{d-1}+\ldots+q+1$ points, d'un ensemble $H$ de $q^{d}+q^{d-1}+$ $\ldots+q+1$ hyperplans. Un hyperplan contient $q^{d-1}+\ldots+q+1$ points, un point est contenu dans $q^{d-1}+\ldots+q+1$ hyperplans. Deux hyperplans se coupent en $q^{d-2}+\ldots+q+1$ points. De même deux points sont communs à $q^{d-2}+\ldots+q+1$ hyperplans.

Il est avantageux de confondre $\mathcal{E P}$ et sa matrice d'incidence $E P(H, E)$. Un espace affine s'obtient, à partir d'un espace projectif fini, en supprimant un hyperplan (on dit alors que c'est l'hyperplan de l'infini) et les points de celui-ci. Un espace affine $\mathcal{A}$ a donc $q^{d}$ points, et $q^{d}+q^{d-1}+\ldots+q$ hyperplans. Un hyperplan a $q^{d-1}$ points (l'hyperplan correspondant de l'espace projectif avait $q^{d-2}+\ldots+q+1$ points dans l'hyperplan de l'infini), deux hyperplans sont ou bien parallèles ou bien se coupent en $q^{d-2}$ points. Les hyperplans parallèles d'une direction donnée

1 Laboratoire d'Informatique Fondamentale, UMR 6166, Université de la Mediterranée, Faculté des sciences de Luminy, 163 avenue de Luminy, 13288 Marseille, France;

e-mail : Anglada, maurras@lif.univ-mrs.fr 
partitionnent l'ensemble des points, il y a donc $q$ hyperplans parallèles d'une direction donnée. On s'intéresse ici aux fonctions caractéristiques des hyperplans d'un espace projectif fini et d'un espace affine fini. Pour une hyperplan $h$ de $H$ son vecteur caractéristique $f$ est un vecteur de $\mathbb{Q}^{E}(\mathbb{Q}$ le corps des rationnels) tel que :

$$
\forall i \in h, f_{i}=1 \text {, et } \forall i \notin h, f_{i}=0 .
$$

La propriété de partition de l'ensemble des points par $q$ hyperplans parallèles à une direction n'est rien d'autre que la traduction de : par un point il passe un (hyper)plan parallèle à une direction donnée.

D'autre part il faudra éviter la confusion entre les hyperplans de l'espace affine $\mathcal{A}$ et ceux du polyèdre $P$ dont les sommets sont les vecteurs caractéristiques des hyperplans de $\mathcal{A} ! .$.

\subsection{QuELQUES PROPRIÉTÉS ÉLÉMENTAIRES}

Soit $M$ une matrice carrée de taille $n$ de la forme $a I+b E$ où $I$ est la matrice identité (diagonale principale de 1 ) et $E$ la matrice dont tous les éléments valent 1 , son inverse est de la forme $c I+d E$. En effet formons le produit de ces deux matrices :

$$
(a I+b E)(c I+d E)=I .
$$

Après avoir remarqué que $E^{2}=n E$, on obtient, $a c I+(a d+b c+n b d) E=I$, d'où : $c=\frac{1}{a}, d=\frac{-b c}{a+n b}=\frac{-b}{a(a+n b)}$. Une condition suffisante d'inversibilité de $M$ est donc $a \neq 0$ et $a \neq-n b$. Ces conditions sont aussi nécessaires, $E$ n'est pas régulière et si $a=-n b$ la somme des colonnes est nulle.

Soit à présent $M$ une matrice carrée de taille $n p$ décomposée en sous-matrices de taille $p$, les sous-matrices de la diagonale principale étant du type précédent, les autres un multiple de $E$. Soit $J$ la matrice (de matrices) dont la diagonale est $I, K$ celle dont la diagonale est $E, L$ celle dont toutes les sous-matrices sont $E$. On a :

$$
J^{2}=J, J K=K, J L=L, K^{2}=p K, K L=L K=p L, L^{2}=n p L
$$

Soit $M=a J+b K+c L$. Montrons que son inverse est de la même forme, soit $a^{\prime} J+b^{\prime} K+c^{\prime} L$. Pour cela formons le produit de ces deux matrices.

$$
(a J+b K+c L)\left(a^{\prime} J+b^{\prime} K+c^{\prime} L\right)=J .
$$

On obtient :

$$
a a^{\prime} J+\left(a b^{\prime}+b a^{\prime}+p b b^{\prime}\right) K+\left(a c^{\prime}+c a^{\prime}+p\left(b c^{\prime}+c b^{\prime}\right)+n p c c^{\prime}\right) L=J
$$

d'où :

$$
a a^{\prime}=1,(a+p b) b^{\prime}+b a^{\prime}=0,(a+p b+n p c) c^{\prime}+c a^{\prime}+p c b^{\prime}=0,
$$


d'où :

$$
a^{\prime}=1 / a, b^{\prime}=-b / a(a+p b), c^{\prime}=-(c / a-p b c / a(a+b p)) /(a+p b+n p c) .
$$

Comme précédemment on a :

Proposition 1. Une CNS pour que $M$ soit régulière est que $a \neq 0$ et $a+p b \neq 0$ et $a+p b+n p c \neq 0$.

Démonstration. En effet supposons que $n$ et $p$ sont supérieurs à 1 , sinon on est ramené au cas précédent. Au vu des relations précédentes il est évident que ces conditions sont suffisantes.

Inversement si $a=0$, les $p$ premières lignes sont linéairement dépendantes (elles sont identiques).

Si $a+p b=0$ la somme des $p$ premières lignes est une ligne dont les éléments sont égaux à $p c$. Ceci est vrai aussi pour les $p$ lignes suivantes. En conséquence les $2 p$ premières lignes sont linéairement dépendantes.

Si $a+p b+n p c=0$, la somme des lignes est nulle.

Remarque 1. On pourrait réitérer la construction de $M$, en considérant des matrices dont les matrices diagonales sont de la forme précédente, on aurait un résultat analogue.

\section{ENVELOPPE CONVEXE DES HYPERPLANS D'UN ESPACE PROJECTIF FINI}

Les $q^{d}+q^{d-1}+\ldots+q+1$ vecteurs caractéristiques des hyperplans d'un espace projectif fini sont linéairement indépendants. Pour s'en convaincre considérons la matrice $A(D, E)$ dont les lignes sont ces vecteurs. On remarque que le produit $M=$ $A A^{t}$ de cette matrice par sa transposée, est la matrice $\left(q^{d-1} I+\left(q^{d-2}+\ldots+1\right) E\right)$, avec $n=q^{d}+q^{d-1}+\ldots+q+1$. Cette matrice est donc régulière et donc $A$ l'est aussi.

Il est clair, dans ces conditions que l'enveloppe convexe de ces vecteurs est un simplexe de $\mathbb{Q}^{E}$ contenu dans la variété définie par $\sum_{e \in E} x_{e}=q^{d-1}+q^{d-2}+\ldots+$ $q+1$.

Remarquons que le vecteur caractéristique de tout hyperplan $h^{\prime} \neq h$ satisfait l'égalité :

$$
\sum_{e \in h} x_{e}=q^{d-2}+\ldots+q+1
$$


par conséquent :

Théorème 1. L'enveloppe convexe des vecteurs caractéristiques des hyperplans d'un espace projectif fini est définie par:

$$
\begin{aligned}
& \sum_{e \in E} x_{e}=q^{d-1}+q^{d-2}+\ldots+q+1, \\
& \sum_{e \in h} x_{e} \geq q^{d-2}+\ldots+q+1, \forall h \in H .
\end{aligned}
$$

Bien entendu, la forme de ces dernières relations n'est pas unique. Cependant il est intéressant de remarquer dans cette expression une relation d'autopolarité du cône défini par ces dernières relations :

$$
\sum_{e \in h} x_{e} \geq q^{d-2}+\ldots+q+1
$$

\section{ENVELOPPE CONVEXE DES HYPERPLANS \\ D'UN ESPACE AFFINE FINI}

Comme précédemment, les vecteurs caractéristiques des hyperplans de l'espace affine satisfont l'égalité $\sum_{e \in E} x_{e}=q^{d-1}$.

On a vu que les hyperplans d'un espace affine $E$ sont partitionnés en $q^{d-1}+$ $q^{d-2}+\ldots+q+1$ blocs de $q$ hyperplans parallèles.

Définition 1. Appelons tangle $t$ un ensemble de $q^{d-1}+q^{d-2}+\ldots+q+1$ hyperplans tel que $t$ contienne exactement un hyperplan par bloc d'hyperplans parallèles. Il y a donc $q^{q^{d-1}+q^{d-2}+\ldots+q+1}$ tangles. $\grave{A}$ un tangle $t$ on peut faire correspondre une inégalité $t_{E} x_{E} \geq \beta$ avec pour $e \in E, t_{e}$ défini comme le nombre d'hyperplans de $t$ contenant le point $e$, et $\beta=\left(q^{d-1}+q^{d-2}+\ldots+q\right) \times q^{d-2}$.

Proposition 2. Ces inégalités définissent des facettes de l'enveloppe convexe des hyperplans de l'espace affine. Ces facettes contiennent exactement $q^{d}-1$ hyperplans.

Démonstration. Il est facile de vérifier que les $q^{d}-1$ hyperplans n'appartenant pas au tangle $t$ satisfont l'inégalité définie par $t$ à l'égalité. En effet deux hyperplans de l'espace affine sécants ont $q^{d-2}$ points en commun; soit h un hyperplan de l'espace affine n'appartenant pas au tangle $t$, il est sécant avec tous les hyperplans de $t$ n'appartenant pas à sa classe d'hyperplans parallèles, la somme $\sum_{e \in E} t_{e} x_{e}$ vaut donc $\left(q^{d-1}+q^{d-2}+\ldots+q\right) \times q^{d-2}$. Pour un hyperplan appartenant à $t$, la contribution à cette somme de lui-même est son cardinal soit $q^{d-1}$. Les $q^{d-1}+$ $q^{d-2}+\ldots+q+1$ autres hyperplans donnent donc la valeur $q^{d-1}+\left(q^{d-1}+q^{d-2}+\right.$ $\ldots+q) \times q^{d-2}$ au premier membre de cette inégalité.

Montrons à présent que les vecteurs caractéristiques de ces $q^{d}-1$ hyperplans sont linéairement indépendants. Pour ce faire remarquons qu'il y a $q-1$ tels hyperplans 
par bloc $B_{j}$ d'hyperplans parallèles. Considérons leur matrice d'incidence $A$ et effectuons le produit de $A$ par sa transposée $A^{t}$, posons $M=A A^{t}$.

Le produit du bloc de lignes de $A$ indicé par $B_{j}$, par celui des colonnes de $A^{t}$ indicé par $B_{j}$, est une matrice dont les éléments diagonaux sont $q^{d-1}$ et les autres 0 .

Pour $i \neq j$, le produit du bloc de lignes de $A$ indicé par $B_{j}$, par celui des colonnes de $A^{t}$ indicé par $B_{i}$, est une matrice dont tous les éléments valent $q^{d-2}$.

$M$ est donc une matrice d'ordre $n p=\left(q^{d-1}+q^{d-2}+\ldots+q+1\right)(q-1)$, dans les notations de la section 1.1, de la forme $q^{d-1} J-q^{d-2} K+q^{d-2} L$. On a donc $a=q^{d-1} \neq 0, a+p b=q^{d-1}+(q-1)\left(-q^{d-2}\right)=q^{d-2} \neq 0$ et $a+p b+n p c=$ $q^{d-1}+(q-1)\left(-q^{d-2}\right)+\left(q^{d-1}+q^{d-2}+\ldots+q\right) q^{d-2} \neq 0$.

$M$ est donc inversible, les $q^{d}-1$ lignes de $A$ sont donc linéairement indépendantes.

Le polyèdre $P$, enveloppe convexe des vecteurs caractéristiques des hyperplans de l'espace affine qui était de dimension au plus $q^{d}-1$ contient donc bien $q^{d}$ points en position générale, les $q^{d}-1$ précédents et n'importe lequel des points (correspondant aux hyperplans) constituant le tangle $t$ dont on vient de voir qu'ils ne satisfont pas l'inégalité définie par $t$ à l'égalité.

Le plan support de l'inégalité définie par le tangle $t$ définit donc bien une facette de $P$.

Théorème 2. L'enveloppe convexe de ces vecteurs caractéristiques est définie par l'intersection de $q^{q^{n-1}+q^{n-2}+q}$ demi-espaces définis par les tangles.

Démonstration. On a vu que les tangles définissaient des facettes simpliciales (elles contiennent le nombre minimum de sommets) de ce polyèdre $P$, montrons qu'aucun autre ensemble de vecteurs caractéristiques d'hyperplans ne peut définir de facette.

Un tel ensemble, qui n'est pas défini par un tangle, contiendrait un bloc complet d'hyperplans parallèles, et donc tout hyperplan les contenant contiendrait leur barycentre le point $\left(\frac{1}{q}, \ldots, \frac{1}{q}\right)$, qui est dans l'intérieur relatif de $P$. En effet tout hyperplan, différent de l'hyperplan de $P$ (défini par $\sum_{e \in E} x_{e}=q^{d-1}$ ), contenant ce point, contient des sommets de $P$ de part et d'autre de lui, et ne peut donc pas supporter de facette de $P$.

On a aussi démontré :

Théorème 3. Soit E l'ensemble des points d'un espace projectif fini d'ordre $q$ et de dimension $d$ et $h$ un de ses hyperplans. La projection sur $Q^{E \backslash h}$ de ce polytope est donc l'enveloppe convexe des droites de l'espace affine obtenu en prenant $h$ comme hyperplan de l'infini de l'espace projectif.

La projection de ce simplexe d'un espace à $q^{d}+q^{d-1}+\ldots+q+1$ dimensions, est un polyèdre d'un espace à $q^{d}$ dimensions ayant $q^{q^{d-1}+\ldots+q+1}$ facettes.

\section{Quelques PROPRIÉtÉS DE CE POLYTOPE}

Les facettes définies par ces plans sont simpliciales, elles contiennent $q^{d}-1$ points. 
Pour $q>2$ tout couple de sommets de ce polytope est relié par une arête.

En effet tout couple d'hyperplans se retrouve comme sommet de la même facette.

Plus généralement :

Proposition 3. Tout sous-ensemble de $k<q$ sommets de ce polytope défini une $k$-face de ce polytope qui est donc $(q-1)$-neighbourly.

Démonstration. En effet un tel sous-ensemble ne contient pas toutes les droites d'un bloc $B_{j}$, et par conséquent peut être complété pour former une facette.

Ce polytope d'un espace à $n=q^{d}$ dimensions a $q^{d}+q^{d-1}+\ldots+q=\frac{q}{q-1}(n-1)$ sommets et $q^{q^{d-1}+\ldots+q+1}=q^{\frac{n-1}{q-1}}$ facettes.

Pour $d=2$ ce polytope d'un espace à $n=2^{d}$ dimensions a donc $2(n-1)$ sommets et $2^{n-1}$ facettes.

Dans les deux cas, pour un corps donné ( $q$ fixé), ce nombre de facettes est exponentiel en $n$ la dimension de l'espace, il est maximisé pour $n=2^{d}$. En [3] nous avions déjà décrit un polytope du cube unité de $2 n$ sommets ayant $2^{n}$ facettes, il serait intéressant de voir si, à partir du polytope que nous venons de décrire, l'on ne peut pas construire un polytope ayant un nombre de facettes améliorant la borne inférieure du nombre maximum de facettes d'un polytope du cube unité qui est actuellement autour de $3^{n}$. Cette borne a été établie par Fleiner, Kaibel et Rote [1]. Il semble que pour fabriquer un polyèdre de l'hypercube unité de $\mathbb{Q}^{n}$ ayant un très grand nombre de facettes on puisse prendre l'enveloppe convexe de $O\left(2^{n-1}\right)$ sommets de ce cube pris au hasard. Les polyèdres considérés en optimisation combinatoire sont en général très symétriques et on un nombre de facettes non exponentiel. On entend dire, par exemple, que le polyèdre du voyageur de commerce a un nombre de facettes exponentiel, ce qui est peut être vrai, mais non prouvé; la seule chose que l'on sait c'est que ce nombre est exponentiel en le nombre $n$ de sommets du graphe (les inégalités de sous-tour sont déjà en nombre $\left.2^{n-1}\right)$, nombre qui n'est pas exponentiel ni en la dimension $n(n-1) / 2$ de l'espace que l'on considère en général, ni en la dimension $n(n-3) / 2$ [2] de l'espace propre de ce polyèdre. Cette famille de polyèdres, en revanche, a un nombre de facettes exponentiel. On peut espérer qu'en rajoutant les point correspondant aux autres sous-espaces de l'espace affine $\mathcal{A}$ on augmente sensiblement le nombre de facettes du nouveau polyèdre qui cependant resterait régulier (on sait qu'en tout cas on ne diminuerait pas ce nombre car le polyèdre que l'on vient de décrire est une facette de ce nouveau polyèdre).

Remarque 2. Dans le cas où $q=2$, tous les points ayant servis à la construction du tangle $t$ valorise le premier membre de l'inégalité définie par $t$ à $2^{d} 2^{d-2}$. Ces $n-1$ points étant affinement indépendants, on a aussi l'inégalité : $t x \leq 2^{d+1}$. Les $n-1$ points ne définissant pas $t$ définissent un tangle $t^{\prime}$, on a donc l'inégalité : $t^{\prime} x \leq 2^{d+1}$. La première inégalité se déduit de celle définie par le tangle $t^{\prime}$ et de l'égalité : $\sum_{e \in E} x_{e}=2^{d_{1}}$. 
Démonstration. Le second membre de l'inégalité définie par un tangle $t^{\prime}$ vaut : $\left(2^{d}-2\right) 2^{d-2}$, retranchons $\left(2^{d}-1\right)$ fois l'égalité $\sum_{e \in E} x_{e}=2^{d-1}$ de l'inégalité définie par $t$, on obtient :

$$
-t_{E}^{\prime} x_{E} \geq\left(2^{d}-2\right) 2^{d-2}-\left(2^{d}-1\right) 2^{d-1},
$$

soit :

$$
t_{E} x_{E} \leq 2^{d} 2^{d-2},
$$

il s'agit de cette inégalité qui n'est donc pas nécessaire.

\section{RÉFÉRENCES}

[1] T. Fleiner, V. Kaibel and G. Rote, Upper bounds on the maximal number of facets of 0/1-polytopes. Eur. J. Combin. 21 (2000) 121-130.

[2] J.F. Maurras, Some results on the convex hull of the hamiltonian cycles of symetric complete graphs, in Comb. Programming Method Application, Proc. N.A.T.O. advanced institute, edited by B. Roy (1975) 179-180.

[3] J.F. Maurras, An exemple of dual polytopes in the unit hypercube. Ann. Discrete Math. 1 (1977) 391-392.

[4] J.F. Maurras, Convex hull of the edges of a graph and near bipartite graphs. Discrete Math. 46 (1983) 257-265.

[5] J.F. Maurras, $k$-arcs et designs dans les plans projectifs finis. Document interne du GRTC, Marseille (1986).

[6] J.F. Maurras, The Line Polytope of a finite Affine Plane. Discrete Math. 115 (1993) 283-286.

[7] B. Segre, Lectures on Modern Geometry. Edizioni Cremonese, Roma (1961).

To access this journal online: www.edpsciences.org 